\title{
Unterwegs zur 08/15-Schule? Wider die Instrumentalisierung der Erziehungswissenschaft durch die Bildungspolitik
}

\section{Walter Herzog}

\author{
No reasonable person is against accountability \\ that enhances the quality of education. \\ Robert L. Linn (2003)
}

Die Schweiz ist im Begriff, ihr Schulsystem radikal zu erneuern. Und zwar ohne dass eine nennenswerte Diskussion geführt würde. Erstaunlich ist insbesondere die Abstinenz der Wissenschaft, die sich fast widerstandslos in die Rolle schickt, die ihr von der Politik zugedacht wird. Dabei stehen Werte auf dem Spiel, deren Verlust nicht widerstandslos hingenommen werden kann. Die folgenden Ausführungen sind aus der Besorgnis entstanden, dass die aktuelle Reform des schweizerischen Schulsystems der Erziehungswissenschaft und Bildungsforschung Schaden zufügen könnte, indem sie in eine Position gedrängt werden, die ihre Unabhängigkeit gefährdet und sie ihrer kritischen Funktion beschneidet.

Ich beginne mit einer knappen Darstellung der laufenden Reformen, wobei ich mich auf den obligatorischen Schulbereich beschränke (1). Danach frage ich nach Herkunft und Bedeutung eines Kernbegriffs der aktuellen Schulreform (2), suche nach Differenzen zwischen industriellen und Bildungsstandards (3) und diskutiere, was der Anspruch auf Messung von Standards beinhaltet (4). Nach einer kritischen Auseinandersetzung mit drei Schwachstellen der aktuellen Reformpolitik (5), schliesse ich mit einigen Bemerkungen zur Lehrerprofessionalität, die nach meiner Beurteilung unter einem ähnlichen Druck steht wie die Erziehungswissenschaft (6).

\section{Eine Reform ohne theoretische Grundlagen?}

Vor ein paar Jahren hat die Schweizerische Konferenz der kantonalen Erziehungsdirektoren (EDK) ein Reformprojekt lanciert, das die obligatorische Schule landesweit vereinheitlichen soll. Geplant ist, die Kantone auf ein neues Schulkonkordat zu verpflichten, das neben einigen anderen Punkten in den 
wichtigsten Fächern Mindest- bzw. Basisstandards festlegt, die am Ende des zweiten, sechsten und neunten Schuljahres überprüft werden sollen. ${ }^{1}$ Die Entwicklungsarbeiten zu diesen Standards, die als Leistungsstandards (sstudent performance standards bzw. (academic achievement standards) definiert sind, laufen unter dem Projektnamen ‘HarmoS〉 (Harmonisierung der obligatorischen Schule). Teil des Projekts ist ein gesamtschweizerisches Bildungsmonitoring, das die EDK zusammen mit dem Bund realisieren will und als dessen Funktion unter anderem die bessere "Steuerung» des schweizerischen Bildungssystems angegeben wird (EDK, 2006, S. 28ff.; SKBF, 2006, S. 6). Ob und wie weit die nationalen Bildungsstandards ihre Funktion als «Steuerungsinstrumente» (EDK, 2007a, S. 3) erfüllen, soll im Rahmen dieses Bildungsmonitorings überprüft werden. Für die (obligatorische) Schule heisst dies, dass ihre Leistungsfähigkeit in Zukunft «am Erreichen der Standards durch die Schülerinnen und Schüler gemessen werden (soll)» (EDK, 2006, S. 31).

Der Erziehungswissenschaft und Bildungsforschung weist das HarmoS-Projekt eine begrenzte, rein instrumentelle Rolle zu. Wissenschaft und Forschung sollen bei der Entwicklung der Bildungsstandards mithelfen und garantieren, dass die mit den Standards verbundenen Kompetenzmodelle korrekt erarbeitet werden. Darüber hinaus soll die Wissenschaft dafür sorgen, dass durch das Bildungsmonitoring jene Daten eingeholt werden, welche die Verwaltung braucht, um das hoch gesteckte Ziel der besseren Steuerung des Bildungssystems zu erreichen. Die Rede ist von «einem systematischen und kontinuierlichen, wissenschaftlich gestützten Monitoring über das gesamte schweizerische Bildungssystem» (EDK 2006, S. 28). Dazu gehört, dass für die Kompetenzen, die den Bildungsstandards zugrunde liegen, Messmodelle und Erhebungsinstrumente entwickelt werden, die verlässlich überprüfen lassen, welches Bildungsniveau die Schülerinnen und Schüler erreicht haben.

Die Aufgabe der Wissenschaft wird als reine Dienstleistung verstanden. Sie soll der Politik helfen, ihre Ziele im Sinne einer «evidence-based policy» (EDK 2006, S. 28, 30) zu verwirklichen. Dieses Politikverständnis ist aus der Medizin herausgewachsen, wo das Modell einer forschungsbasierten klinischen Entscheidungsfindung (evidence-based medicines) schon seit längerem in Gebrauch ist (Marston \& Watts, 2003, S. 146f.). Der Nutzen von Wissenschaft wird in der Produktion von Fakten gesehen, die der Politik klare Entscheidungen erlauben. Gefordert wird «ein Maximum an gesichertem (wissenschaftlich und statistisch abgestütztem) Wissen» (EDK, 2006, S. 30 - Hervorh. W.H.). Das Bildungsmonitoring bindet die Wissenschaft in einen Zyklus ein, der mit Fragen und Bedürfnissen der Politik beginnt und über die Sammlung, Aufbereitung und Interpretation relevanter Daten zu Steuerentscheidungen und neuen Fragen der Politik führt (EDK 2006, S. 30; SKBF, 2006, S. 7). ${ }^{2}$ Dadurch soll erreicht werden, dass die Politikerinnen und Politiker ihre Massnahmen nicht länger «auf Grund von Vermutungen oder Überzeugungen, sondern auf Grund von Daten und erwiesenen Kausalitäten» (Kull, 2007, S. 11 - Hervorh. W.H.) ergreifen. 
Die Ausblendung der kritischen Funktion von Wissenschaft ist politisch leicht nachvollziehbar, geht es doch darum, einem ambitiösen Projekt Legitimation zuzuführen, und das scheint am ehesten zu gelingen, wenn man die Wissenschaft als Hüterin der Wahrheit für sich gewinnen kann. Wenn die Wahrheit zudem auf Evidenz reduziert wird, kann auch der anstrengenden Kontroverse um Genese und Geltung von (wissenschaftlicher) Erkenntnis ausgewichen werden. Bereits Rousseau (1762/1971, S. 157) bediente sich dieser Strategie, als er im «Emil» behauptete, sich ausschliesslich auf die Erfahrung zu berufen und dem Theoretisieren «so wenig Raum wie möglich» (ebd., S. 263) zu geben. "Ich stütze mich nicht auf das, was ich erdacht, sondern auf das, was ich gesehen habe» (ebd., S. 263 - H ervorh. W.H.). Wenn uns die Wahrheit gleichsam vor Augen liegt, dann ist die Politik wahrlich gut beraten, die Wissenschaft in ihr Boot zu holen.

Nur gibt es eine solche Wahrheit nicht! Reine Fakten sind uns ebenso wenig zugänglich wie reine Gedanken. «There are no ultimate sources of knowledge. ... every source, every suggestion, is open to critical examination» (Popper, 1969, S. 27). Eine unbefleckte Erkenntnis ist unter irdischen Verhältnissen genauso wenig möglich wie eine unbefleckte Empfängnis. All unser Wissen ist theoretisch imprägniert; schon in unsere Sinnesorgane sind antizipierende Theorien eingebaut (Popper, 1974, S. 164f.). Die «Kübeltheorie des Geistes» (Popper), die es der Politik erlauben würde, die Wissenschaft auf das Einsammeln von neutralen Daten zu verpflichten, ist eine reine Fiktion.

Die Wahrheit einer Erkenntnis ist eine Sache ihrer Begründung. "Whether a naturally occurring phenomena or a research artifact, the stuff of the world only becomes (evidences when (it) is constituted and inserted into a research practice and then deployed in the framework of an argument» (Marston \& Watts, 2003, S. 152). Dabei spielen Annahmen über die Struktur der Wirklichkeit und das angemessene Vorgehen bei ihrer Erforschung eine wesentliche Rolle. Anzunehmen, es gebe so etwas wie einen «goldenen Standard» der wissenschaftlichen Methode, wäre genauso naiv, wie zu glauben, Wahrheit beruhe auf blosser Evidenz. Den Kern der wissenschaftlichen Rationalität bilden nicht Evidenzen, sondern Argumente: "... what is key is how the evidence is used in the course of argumentation» (Phillips, 2006, S. 25). Das aber heisst, dass «... no evidence claim underpinning evidence-based policy arguments can be considered detached, value free, and neutral» (Marston \& Watts, 2003, S. 157).

Wenn es wissenschaftliche Erkenntnis ohne theoretische Annahmen nicht gibt, dann kann der Versuch der Indienstnahme der Forschung durch die Politik nur bedeuten, dass von der Theorielosigkeit der Reform des schweizerischen Bildungssystems abgelenkt werden soll. Das Beispiel der USA, die schon länger mit ähnlichen Reformen experimentieren, zeigt, dass diese Analyse nicht falsch ist. So schreibt Elmore (2004), nachdem er verschiedene Mängel von leistungsbasierten Rechenschaftssystemen (‘accountability systems)) referiert hat, Verbesserungen hätten als erstes zu berücksichtigen, «... that individual and collective sta- 
kes should be based on defensible, empirically based theories about what it is possible to accomplish on measured performance within a given period of time» ( $\mathrm{S}$. 290 - Hervorh. geändert, W.H.). Was stattdessen geschehe, sei eine Ausrichtung der staatlichen Politik an Belohnungen und Bestrafungen, die willkürlich festgelegt würden und in nicht wenigen Fällen sowohl pädagogisch wie auch psychometrisch unsinnig seien. "No accountability system currently has such a working theory at any level of specificity that is useful as a guide to action for school administrators or teachers" (ebd., S. 291). Im Umlauf sind implizite Theorien, die der Öffentlichkeit von der Politik aufoktroyiert werden: "The central fact of accountability systems as they presently exist is that they are political artifacts crafted out of relatively superficial and underspecified ideas to meet the demands of political action» (ebd., S. 295).

Die Amputation der Wissenschaft um ihre theoretische und kritische Dimension bedeutet, dass die Annahmen über die Struktur der (pädagogischen) Wirklichkeit, die den laufenden Schulreformen zugrunde liegen, implizit bleiben. Welcher Art diese Annahmen sind, wird ersichtlich, wenn man in Rechnung stellt, dass unter Bildungsstandards vor allem Leistungs- bzw. Ergebnisstandards verstanden werden (Böttcher, 2006). Das gilt für die USA genauso wie für die Schweiz. Angenommen wird ein simples Verstärkungsmodell, wonach jenes Verhalten häufiger auftritt, das belohnt wird, und jenes seltener, das bestraft wird. Etwas ausführlicher umschreibt Elmore (2004) die heimliche Theorie der standardbasierten Schulreform: «Performance-based accountability systems operate on the theory that measuring performance, when coupled with rewards and sanctions ... will cause schools and the individuals who work in them, including students, teachers, and administrators, to work harder and perform at higher levels ... The idea is appealingly simple: design an incentive structure that rewards students for engaging their energy in learning academic content at high levels, teachers for teaching a broad range of students more effectively, and schools for organizing themselves to manage instruction more effectively» (S. 277f.). ${ }^{3}$

Geht die Beschneidung der Wissenschaft um ihre theoretische Dimension demnach mit einem heimlichen Bekenntnis der Politik zu einem technologischen Erziehungsverständnis einher? Wir wollen uns mit der Bejahung dieser Frage noch etwas zurückhalten und vorerst einen der zentralen Begriffe der laufenden Schulreform näher untersuchen: den Begriff der Bildungsstandards.

\section{Von der handwerklichen zur industriellen Fertigung}

Um zu erfahren, was es mit Bildungsstandards auf sich hat, sollte ein Abstecher zur ISO, der «International Organization for Standardization», nicht abwegig sein. Auf deren Homepage ${ }^{4}$ kann man lesen, Standards würden einen wesentlichen Beitrag zu den meisten Aspekten unseres Lebens leisten, auch wenn die- 
ser Beitrag oft unsichtbar bleibe. Es sei die Abwesenheit von Standards, die uns ihre Bedeutung bewusst mache. So gesehen wäre die Diskussion um Standards im Bildungswesen ein Zeichen dafür, dass es in Schule und Unterricht bisher keine Standards gegeben hat. Das dürfte allerdings kaum zutreffen, wie wir noch sehen werden (vgl. Abschnitt 3).

Standards sind Instrumente der Normierung. Das zeigt allein schon der französische Name für ISO: «Organisation internationale de normalisation». Noch deutlicher wird die Zielsetzung, wenn die ISO ihren Namen erläutert, der nämlich nicht eine Abkürzung in einer bestimmten Sprache und auch kein Akronym darstellt, sondern eine Referenz an das griechische Wort «isos» darstellt, das "gleich» bedeutet. Standardisierung ist demnach Normierung im Sinne von Gleich- bzw. Vergleichbarmachung. Wobei die Reichweite des Vergleichs global ist: Die Standards der ISO haben internationale Gültigkeit. Seit Anfang des 20. Jahrhunderts bestehen solche Standards in der Industrie, vor allem in der Maschinen- und Elektrotechnik. Noch 1947, im Gründungsjahr der ISO, ging es allein um die Koordination und Vereinheitlichung von industriellen bzw. technischen Standards.

Die Abkunft der Standardisierung von der industriellen Fertigung lässt sich am vielleicht berühmtesten Beispiel für einen Standard illustrieren: der 08/15Norm. In seiner vollen Bezeichnung heisst der Standard «M.G. 08/15». «M.G.» steht für Maschinengewehr, «08» für das Jahr 1908 und «15» für die verbesserte Version des Standards, wie er 1915 eingeführt wurde. Erfinder des M.G. 08 war Sir Hiram Stevens Maxim, dessen automatische Waffe nach zehnjähriger Erprobungsphase 1908 in staatliche Produktion ging (Berz, 2001, S. 582ff., 699ff.).

Mit dem Ausbruch des Ersten Weltkriegs genügte die handwerkliche Fertigungstechnik der Waffenschmiede der militärischen Nachfrage nicht mehr. Die Waffenproduktion musste intensiviert werden. Das war nur möglich, indem Betriebe mobilisiert wurden, die mit der Herstellung von Waffen bisher nichts zu tun hatten. Fabriken, die Rechenmaschinen, Fahrräder, Nähmaschinen, Teppiche, Scheren etc. herstellten, produzierten Teile, die sie dank besonderem Knowhow mit hoher Qualität anfertigen konnten. Die Teile wurden dann zentral zum Maschinengewehr zusammengesetzt, ein Vorgehen, das man «unterteilte Fertigung» nannte.

Im Vergleich zur handwerklichen musste die «unterteilte» Fertigung von Kriegsgeräten völlig neu organisiert werden. Während der Kern des Handwerks in der ganzheitlichen Herstellung eines Produkts liegt, das aus Teilen hervorgeht, die nicht vorweg aufeinander abgestimmt sind und dementsprechend durch Feilen, Schleifen und Schmirgeln bearbeitet werden müssen, geschieht die industrielle Fertigung nicht mehr von Hand. Aus Technik wird Technologie (Böhme 1993, S. 453ff.). Die Produktion wird durch Maschinen ersetzt, was nur geht, wenn die Einzelteile normiert sind. Erforderlich ist eine einheitliche Präzision, die von Plänen und Konstruktionszeichnungen garantiert wird, in denen Toleranzwerte angegeben sind, die vor Ort überprüft werden können (Berz, 2001, S. 
65ff.). Die Beschreibung der Einzelteile setzt den Standard ihrer Fertigung. Was innerhalb des Toleranzbereichs liegt, ist gute Qualität, alles andere ist Ausschuss und kann bereits von der Zulieferfirma aufgrund der Messung des Outputs ausgesondert werden. Nicht lokales Einpassen wie beim Handwerk, sondern kontextfreies Messen ist die Basis der industriellen Fertigung.

Damit lässt unser Abstecher zur ISO nicht nur etwas klarer sehen, was mit Standards gemeint ist, wir sehen zugleich, wie die Begriffe Qualität und Output, die in der aktuellen bildungspolitischen Diskussion ebenfalls eine prominente Rolle spielen, mit dem Standardbegriff zusammenhängen.

\section{Von der Input- zur Outputsteuerung}

Trotz einer langen Tradition von pädagogischen Produktionsmetaphern und phantasien (Herzog, 2002, S. 40ff., 2007a), werden wir kaum geneigt sein, Erziehung und Unterricht mit der Herstellung eines Maschinengewehrs zu vergleichen. Und trotzdem ist nicht von der Hand zu weisen, dass die Diskussion von Bildungsstandards nicht einer handwerklichen, sondern einer industriellen Logik folgt. Das lässt sich leicht zeigen, wenn wir uns fragen, was denn bisher für die Qualität von Schule und Unterricht garantiert hat. Es sind nicht zuletzt Gesetze, Verordnungen, Lehrpläne und Prüfungsreglemente, die vorgeben, wie Schule zu gestalten ist, die gewährleisten, dass eine gewisse Einheitlichkeit der «Produktionsverhältnisses besteht, und die dafür sorgen, dass die (Produktionsmittel) zwischen den Schulen angehend gleich verteilt sind. Auch das Prinzip der Jahrgangsklasse, die Übertrittsverfahren und die Dauer der Schulpflicht geben vor, wie Schule zu gestalten ist und sind insofern Formen der Standardisierung von schulischer Bildung. In Schule und Unterricht ist es schon immer auch darum gegangen, vergleichbare Verhältnisse zu schaffen, Ziele und Mittel festzulegen sowie die Organisation der Lehr- und Lernprozesse zu normieren, d.h. die Bedingungen gleich bzw. vergleichbar zu machen, unter denen Schule stattfindet (Aldrich, 2000; Oelkers, 2004).

Allerdings haben wir es bei den genannten Normen und Regeln fast ausnahmslos mit Vorgaben zu tun, die den «Inputbereich` von Schule betreffen. Es sind Vorschriften im wörtlichen Sinn, die den Rahmen festlegen, innerhalb dessen Schule zu halten ist. Es sind weder Produktionsanleitungen, die den Fertigungsprozess im Detail regeln, noch Messvorschriften, welche die Qualität des Produkts exakt überprüfen liessen. Genau hier liegt die Differenz zwischen den Standards, denen die Schule bisher gefolgt ist, und den Bildungsstandards, die ihr neu auferlegt werden. Wie für industrielle Standards (vgl. Abschnitt 2), ist für Bildungsstandards die Überwindung der Beschränkung auf lokale Vergleiche charakteristisch. Jedoch geht es nicht um einen umfassenden, globalen Blickwinkel, aber immerhin um eine nationale Perspektive (EDK, 2004, 2006; Klieme et al., 2003). Angestrebt wird «eine Richtschnur, die national festsetzt, welches die 
minimalen Anforderungen der Volksschule sind und wann genau diese als erreicht gelten» (Maradan 2005, S. 8 - Hervorh. W.H.). Wie PISA reaktiviert die Einführung von Bildungsstandards ein fragwürdiges Nationendenken.

Damit haben wir zwei Kriterien gefunden, in denen sich die aktuelle Diskussion von Qualitätsansprüchen unterscheidet, die schon immer an die Schule gerichtet wurden. Erstens beziehen sich Bildungsstandards auf den schulischen Output, und zweitens legen sie fest, wie der Output im nationalen Rahmen ausfallen soll. Insofern unterscheiden sich Bildungsstandards kaum von einer ISONorm. Das gilt auch für die Steuerungsfunktion von Bildungsstandards, die ein drittes Vergleichskriterium abgibt. Bildungsstandards sind «normative Vorgaben für die Steuerung von Bildungssystemen», heisst es im Klieme-Bericht (Klieme et al., 2003, S. 32), an den sich das HarmoS-Projekt so eng anlehnt, dass ihn die EDK gleich ins Französische übersetzen liess. Indem die Schule nicht daran gemessen wird, ob sie den behördlichen Vorschriften folgt, sondern inwiefern sie ihren Auftrag erfüllt (EDK, 2004, S. 7), wird ihr eine neue Form der Verwaltungsführung auferlegt, die sich «Outputsteuerung) nennt.

\section{Thorndike lässt grüssen}

Damit bestätigt sich, dass Bildungsstandards weniger Ausdruck eines pädagogischen als eines politischen Interesses sind. Dessen Ziel ist die verstärkte Einflussnahme auf das schweizerische Bildungssystem, das nicht nur in seinen Leistungen, sondern auch in seinen Strukturen, Zielen, Inhalten und Methoden vereinheitlicht werden soll (EDK, 2004, S. 4, 2006; Maradan \& Mangold, 2005; Mangold, Rhyn \& Maradan, 2005, S. 178f.). Dass man sich dabei von der Logik der industriellen Fertigung leiten lässt, ist offensichtlich. Die über die Wirkungen von Schule definierten "schweizerischen Bildungsstandards» setzen gleichsam am Ende der Fertigungsschiene an; content- und opportunity-to-learnstandards, die sich auf curriculare Inhalte und didaktische Ressourcen beziehen würden, werden explizit ausgeschlossen (Maradan \& Mangold, 2005, S. 4).

Um die Qualität eines Outputs garantieren zu können, muss er gemessen werden (vgl. Abschnitt 2). Das wirft die Frage auf, ob an Schulen bisher nicht gemessen wurde. Aber was anderes als ein Messinstrument ist eine Notenskala? Und ist ein Zeugnis nicht ebenfalls das Ergebnis eines Messvorganges? Die Verfechter von Bildungsstandards geben zur Antwort, dass die Messoperationen an unseren Schulen nicht reliabel und valide seien. Allein schon die Messkriterien, wie sie durch die Lehrpläne vorgegeben werden, seien zu unbestimmt, würden zu viel der Interpretation anheim stellen und seien aufgrund schöngeistiger Formulierungen nicht operationalisierbar. Wo der Stoff trotzdem geprüft werde, da seien die Massstäbe zwischen den Lehrpersonen und den Schulen ungleich, weshalb die Schulnoten einen geringen Aussagewert hätten. Die Rede ist von «subjektiven und heterogenen Bewertungen von Schülerleistungen» (EDK, 2006, S. 
24). Was Schülerinnen und Schüler tatsächlich können, bleibe offen (EDK, 2004, S. 5). 5

Der Vorzug von Bildungsstandards wird darin gesehen, dass sie die schulische Beurteilung von lokalen (handwerklichen) Normen ablösen lassen. Dank der Entwicklung von Messmodellen soll eine metrische Skalierung schulischer Leistungen möglich werden. Angeschlossen wird an das Credo von Thorndike (1921), der den Glauben an die Wohltaten der Psychometrie wie folgt auf den Punkt brachte: «Education ... will profit by measurements of human nature and achievement as mechanical and electrical engineering have profited by using the foot-pound, calorie, volt, and ampere» (S. 371). Dem Dunstkreis der Waffenproduktion sind wir offensichtlich noch nicht entronnen.

Gemessen wird nun nicht etwa schulisches Wissen, gemessen werden vielmehr Kompetenzen, «welche die Schule ihren Schülerinnen und Schülern vermitteln muss, damit bestimmte zentrale Bildungsziele erreicht werden» (Klieme et al., 2003, S. 19). Kompetenzen sind Dispositionen, die Personen befähigen, «konkrete Anforderungssituationen eines bestimmten Typs zu bewältigen» (ebd., S. 72). Demgemäss wird im HarmoS-Projekt für jeden der vier Fachbereiche, auf die man sich geeinigt hat - Erstsprache bzw. Unterrichtssprache, Fremdsprachen, Mathematik und Naturwissenschaften -, ein Messmodell entwickelt, «welches Abstufungen und Entwicklungsverläufe von Kompetenzen sichtbar [!] macht» (EDK, 2004, S. 9). Ich übergehe die Frage, wie es kommt, dass die Schule nach Meinung der EDK nicht mehr wissens-, sondern kompetenzenorientiert unterrichten soll, und hake stattdessen beim Stichwort der Sichtbarmachung ein, das mir erlaubt, auf das Anliegen der «Steuerung` zurückzukommen.

Obwohl Kompetenzmodelle entwickelt werden, um Leistungen von Individuen zu messen, dienen Bildungsstandards nicht der Einflussnahme auf individuelle Lernprozesse. Vielmehr stehen sie im Dienste der Qualitätssicherung auf der Systemebene. Bildungsstandards kommt eine ähnliche Funktion zu wie den PISA-Studien. Denn auch diese dienen nicht dem Vergleich von Einzelschulen oder individuellen Lehrer- oder Schülerleistungen. Dies schon allein deshalb nicht, weil es die Güte der Tests nicht zuliesse, verlässliche Aussagen über individuelle Leistungen zu machen. Dem entspricht, wenn die EDK schreibt, es gehe nicht darum, «die Ergebnisse des Projekts HarmoS für die Evaluation der Lehrpersonen oder die Beurteilung und Selektion der Schülerinnen und Schüler zu nutzen, sondern um die Steuerung des Schulsystems» (EDK, 2004, S. 13). Dass es darum geht - um Systemmonitoring und Systemsteuerung - wird auch daran erkennbar, dass die Standards am Ende der 2., 6. und 9. Klasse überprüft werden, also immer dann, wenn ein Abschnitt einer schulischen Karriere zu Ende geht. Wenn es um etwas anderes ginge, so insbesondere um die Förderung der einzelnen Schülerinnen und Schüler, dann wären dies die falschen Zeitpunkte.

Angesichts dieser klaren Positionierung der EDK ist schwer zu verstehen, weshalb gewisse Kantone anderes im Schilde führen. ${ }^{6}$ Bildungsstandards sollen nicht nur zur Systembeobachtung, sondern auch zur vermeintlichen Objektivie- 
rung von Selektionsentscheidungen genutzt werden. Diesen Kantonen wäre anzuraten, die Erfahrungen zur Kenntnis zu nehmen, die man andernorts mit solchen Kurzschlüssen zwischen System- und Individualebene gemacht hat. So zum Beispiel in den USA, wo man die Verwendung von Tests, die über die schulische Karriere entscheiden, «High-Stakes Testing» nennt. Dazu gibt es mittlerweile eine wachsende kritische Literatur, die zeigt, dass die Tests nicht messen, was sie zu messen vorgeben, schwache Schülerinnen und Schüler noch schwächer werden, der Unterricht sich an den Outputmessungen orientiert ("teaching to the test»), die Lehrerzentriertheit des Unterrichts (wieder) zunimmt und Lehrer wie Schüler demotiviert werden (Amrein \& Berliner, 2002; Böttcher, 2006; Hargreaves, 2003; Heubert \& Hauser, 1999; Kohn, 2000; Lam, 2004; Lind, 2004; Linn, 2000, 2003; McNeil, 2000; Nichols, Glass \& Berliner, 2006; Orfield \& Kornhaber, 2001; Sheldon \& Biddle, 1998). ${ }^{7}$ Diese Literatur lässt nur eine Folgerung zu: Bildungsstandards dürfen nicht für Notengebung, Zertifizierung und Selektionsentscheidungen missbraucht werden (Klieme et al., 2003, S. 48, 107ff.)!8

\section{Schwachstellen einer technokratischen Reform}

Wenn wir auf die Frage zurückkommen, ob mit der Instrumentalisierung der Wissenschaft für die Zwecke der aktuellen Reform des schweizerischen Bildungssystems ein Bekenntnis zu einem technologischen Erziehungsverständnis einhergeht, so lässt sich eine bejahende Antwort nicht mehr vermeiden. Offensichtlich sind Bildungsstandards auf ein implizites Verständnis von Erziehung und Unterricht zugeschnitten, das dem Produktionsmodell der industriellen Fertigung sehr nahe kommt. Eine politisch motivierte Reform bedient sich einer Sprache, deren Herkunft simple Vorstellungen von pädagogischer Wirksamkeit aktiviert. Wie Shirley und Hargreaves (2006) - mit Blick auf die USA - etwas zynisch bemerken: "Right now, data-driven instruction, results-oriented improvement, and evidence-based education are the watchwords. They show up everywhere ... insisting that instructional practices should be driven by the analysis of student-achievement data as measured by prescribed standardized tests. Of course, data-driven instruction sounds tough and businesslike. No need to actually think about what you're doing, just let the data drive you» (S. 32).

Genau dieses "no need to actually think about what you're doing» steht hier unter Anklage. Wer immer sich mit Daten zu begnügen und auf das Denken zu verzichten vermag, die Wissenschaft ist dazu nicht in der Lage. Daher kann die Erziehungswissenschaft die Rolle, die ihr bei der laufenden Reform des schweizerischen Bildungssystems zugedacht wird, nicht akzeptieren. Im Folgenden möchte ich an drei ausgewählten Beispielen zeigen ${ }^{9}$, dass auf das kritische Potential der Wissenschaft nicht ohne Folgen verzichtet werden kann. In allen Fällen geht es um missachtete Aspekte von Bildung und Erziehung, die Zweifel wecken, ob die von der EDK aufgegleiste Reform auf dem richtigen Weg ist. 


\section{Pädagogische Prozesse sind nicht technisierbar}

Wenn wir unter einer Technologie eine Koppelung von Ursachen und Wirkungen verstehen, die mit vorweg bekannter Wahrscheinlichkeit ein Ziel erreichen lässt, dann fehlt uns das Wissen, um eine Erziehungstechnologie zu begründen. Niemand kann bei einem Schüler oder einer Schülerin vorab sagen, was aus ihm oder ihr werden wird. Selbst wenn alle Einflüsse auf das Kind oder den Jugendlichen bekannt wären oder kontrolliert werden könnten, eine rationale Planung des pädagogischen Outputs in Bezug auf diesen Schüler oder diese Schülerin ist nicht möglich. Seit Luhmann und Schorr (1979) nennt man dies das «Technologiedefizit» der Erziehung, für das sich mindestens drei Argument anführen lassen.

Erstens haben wir es im Falle von Schule und Unterricht mit sozialen Systemen zu tun, die sich nicht in Teile zerlegen lassen, die zusammen ein Ganzes ergeben. Ansatzpunkt der neueren Systemtheorie, wie sie insbesondere von Luhmann (1984) ausgearbeitet wurde, ist die Unterscheidung von System und Umwelt, die operativen Charakter hat und vom Standpunkt abhängig ist, von dem aus die Unterscheidung getroffen wird. Damit wird das Input-Output-Modell, wie es der industriellen Fertigung zugrunde liegt, ausser Kraft gesetzt und durch ein anspruchsvolleres Analyseschema ersetzt. Lehrer und Schüler bilden keine Systemeinheit, in der Information wie in kommunizierenden Röhren zirkulieren könnte. Vielmehr stehen sie gegenseitig im Verhältnis von System und Umwelt. Das Lehren des Lehrers und das Lernen des Schülers sind Operationsmodi, die in verschiedenen Systemen ablaufen und nicht kausal, sondern kommunikativ miteinander verbunden sind (Herzog, 2002).

Zweitens steht in einer pädagogischen Situation nicht ein Subjekt einem Objekt gegenüber. Vielmehr ist der 'Gegenstand der Erziehung seinerseits durch Subjektivität ausgezeichnet. Dem Ich steht nicht ein Es, sondern ein Du gegenüber - wie ‘unmündig) es auch immer sein mag. Gotthard Günther (1991) hat in verschiedenen Arbeiten aufgezeigt, dass die zweiwertige Logik des abendländischen Denkens von dieser Konstellation gesprengt wird. Was wir brauchen, um pädagogische Situationen angemessen zu beschreiben, ist eine mehrwertige Logik. Diese schliesst ein technologisches Verständnis von Erziehung und Unterricht a priori aus, da eine technische Praxis in logischer Hinsicht lediglich auf einer zweistelligen Relation beruht. Die industrielle Fertigung eines Maschinengewehrs ist technologisch vollständig beherrschbar, die Bildung eines Menschen ist es nicht.

Drittens bilden Schulen komplexe Organisationen, die sich nicht im wörtlichen Sinne steuern lassen (O’Day, 2004; Senge, 1997; Specht, 2006). Reformen haben wenig Chancen, wenn sie von den Betroffenen nicht mitgetragen werden (Brügelmann, 2004, S. 421f.). Wenn daher von «Outputsteuerung» die Rede ist, welche die bisherige "Inputsteuerung» des Schulsystems ersetzen soll (vgl. Abschnitt 3), dann wird entweder unklar geredet, oder man weiss nicht, wovon man spricht. Denn steuern lässt sich im technischen Sinn immer nur ein Input, sicher nicht ein Output. Die Herkunft des Begriffs aus der Kybernetik 
zeigt zudem, dass zwischen Steuerung und Regelung zu unterscheiden ist (Ashby, 1985, Teil III). Steuerung impliziert ein Handeln, das entscheidungsbasiert ist, also von einem Menschen ausgeführt wird, während Regelung ein technischer Ablauf ist, der so vor sich geht, dass im Rahmen eines vorgegebenen Sollwerts Abweichungen automatisch kompensiert werden. Die Vorstellung, mittels Standards liessen sich Bildungsprozesse auf ein Niveau der Verlässlichkeit anheben, wie wir es von industriellen Fertigungsprozessen gewohnt sind, ist daher absurd. Und dass mittels Bildungsstandards sogar «besser gesteuert werden kann, was Kinder und Jugendliche lernen» (Huber, 2006, S. 180), ist noch absurder. Das Technologiedefizit der Pädagogik ist nicht überwindbar - auch durch Bildungsstandards nicht. ${ }^{10}$

Ins Grundsätzliche gewendet wäre gerade die Erziehungswissenschaft gut positioniert, um eine doppelte Einsicht zu vermitteln, die Einsicht nämlich, dass wir erstens in einer Welt leben, deren wachsende Komplexität zum grossen Teil von uns selbst erzeugt wird, und dass zweitens unser Nicht-Wissen kein NochNicht-Wissen, sondern ein Nicht-Wissen-Können ist (Herzog, 2002; Kade \& Seitter, 2003). Nicht nur das pädagogische, sondern auch das bildungspolitische Handeln wird sich damit abfinden müssen, dass Eingriffe in soziale Systeme eine rationale Planung im herkömmlichen Sinn nicht zulassen. Insofern kommt die Simplifizierung der pädagogischen Wirklichkeit mittels technologischer Modelle einer Selbstillusionierung gleich, die keinen konstruktiven Beitrag zur Lösung der aktuellen Probleme von Bildung und Erziehung leistet.

\section{Die Schule besteht nicht nur aus output}

Selbst wenn wir dem Argument zuliebe annehmen wollten, Bildungsprozesse liessen sich im Rahmen des Input-Output-Schemas angemessen rekonstruieren, zeigt die nähere Betrachtung, dass dem nicht so ist. Nehmen wir zum Zweck der Illustration die Kriterien einer (guten Schule), die gemäss einer Liste von Fend (1994) unter anderem folgende Elemente umfassen: "Gute Schulen sind solche, in denen sich Lehrer und Schüler über Jahre kennen, in denen gegenseitige Besonderheiten und Eigenheiten wahrgenommen und toleriert werden. ... In guten Schulen passiert etwas; es ist ‘viel los`, Feste werden gefeiert, Ausflüge organisiert, Ausstellungen arrangiert - wobei die jeweilige Vorbereitung wichtiger ist als die Durchführung. ... Gute Schulen sind nicht überbürokratisch, sie verschüchtern die Schüler nicht, sie ersticken Aktivitäten nicht in Regelungen und Ängstlichkeiten. ... In guten Schulen ist eine freundliche, lockere Atmosphäre spürbar, Lehrer sind gerne dort, Schüler fühlen sich wohl und Eltern haben den Eindruck, ihr Kind ... ist dort gut aufgehoben»(S. 18f.).

Interessant an dieser Aufzählung ist, dass die Merkmale nur marginal die Unterrichtsebene betreffen und die Schule eher als Lebenseinheit denn als Produktionsstätte beschrieben wird. An guten Schulen fühlt man sich wohl, und zwar nicht nur als Schülerin oder Schüler, sondern auch als Lehrerin oder Lehrer. Interessant ist zudem, dass sich die Merkmale von guten Schulen kaum als 
Kausalursachen für schulischen Output verstehen lassen. Wenn es gar heisst, die Vorbereitung einer Aktivität sei wichtiger als deren Realisierung, so wird ein Output nicht einmal erwartet. Zwar werden an guten Schulen auch gute Leistungen erbracht, aber viele der von Fend (1994) aufgezählten Merkmale sind an sich wertvoll und nicht weil sie für einen bestimmten Zweck nützen.

Es ist genau dieses Moment des Zwecklosen und an sich Wertvollen, das ausgeblendet wird, wenn Schulen in einer rein zweckrationalen Perspektive im Hinblick auf ihre Produktivität betrachtet werden. Was vergessen geht, sind die atmosphärischen Merkmale von Schule, die vermeintlich nutzlosen Dinge, wie die Freude an einem Fach, das Wohlergehen im Unterricht, die Zufriedenheit mit einer Leistung, der Umgang zwischen Lehrer und Schüler, die Überraschung durch eine unerwartete Antwort, der freiwillige oder unfreiwillige Humor u.ä. All dies lässt sich nur schwer messen und findet in keinem Kompetenzmodell Platz, weil es weder als Output eines Inputs zustande kommt, noch in Form von Kompetenzniveaus darstellbar ist. Zweifellos schliessen Bildungsstandards dies nicht aus. Das Problem ist nur, dass der Fokus, den Bildungsstandards auf den Output legen, die Schule in ein Licht rückt, das die flüchtigen und scheinbar unproduktiven Momente von Schule übersehen lässt.

\section{Bildung kann nicht nur quantitativ bestimmt werden}

Bildungsstandards sind das Kleingeld eines politischen Diskurses, der auf pädagogische Fragen nur mehr ökonomische Antworten sucht. Alle mussten wir in den vergangenen Jahren Wörter dazulernen, die bislang im Bildungswesen unbekannt waren, wie Effizienz, Benchmarking, Best Practice, Humankapital, Globalbudget, Controlling, Leadership etc. Fast alle diese Begriffe stammen aus der Betriebswirtschaftslehre (Dubs, 1996). Auch die Begriffe Qualitätsmanagement und Outputsteuerung gehören dazu. Die aktuelle Schulreform steht im Zeichen der Wirtschaftspolitik, und das gilt uneingeschränkt auch für Bildungsstandards. ${ }^{11}$

Das hat zur Folge, dass über Ziele der (schulischen) Bildung kaum noch diskutiert wird. So ist beim HarmoS-Projekt über die Auswahl der Fächer, für die Bildungsstandards entwickelt werden, keine Debatte geführt worden. Von Seiten der EDK erfährt man nur, dass es sich bei den ausgewählten Fachbereichen um solche «von grundlegender Bedeutung» (EDK 2004, S. 2) handelt. Wofür diese Fachbereiche "von grundlegender Bedeutung» sind, wird nicht gesagt. Stattdessen heisst es, sie würden auch in anderen Ländern bei der Bestimmung von Standards berücksichtigt. Wie wenig Diskussion nötig war, um die Fächer festzulegen, zeigt die Tatsache, dass man sich schon früh darauf geeinigt hat. In den Projektunterlagen wird die Auswahl der Fächer weder als Teil des Projekts dargestellt noch unter dessen Vorarbeiten aufgeführt (Maradan \& Mangold, 2005, S. 6). Entschieden wurde offenbar allein aufgrund der Normativität des Faktischen und der Macht von Gremien. ${ }^{12}$

Dass die Inhalte an Bedeutung verlieren, ist eine direkte Folge des Kompetenzbegriffs, auf den man sich stützt. Wenn Kompetenz funktional als Potential 
gedacht wird, das auf Lebensbewältigung ausgerichtet ist und nicht den Inhalten der Lehrpläne entspricht (Klieme et al., 2003, S. 72ff.), dann brauchen die Letzteren zwar nicht ersetzt zu werden, wie die EDK gerne betont (Maradan \& Mangold, 2005, S. 4; Mangold, Rhyn \& Maradan, 2005, S. 179), für die Messung von Bildungsstandards sind sie aber bedeutungslos. Als rein formales Konzept sind Kompetenzen offen für beliebige Inhalte. Wenn - um ein Beispiel zu geben - als sprachliche Kompetenz gefordert wird, «Texte zu verstehen, zu nutzen und über sie zu reflektieren» (OECD, 2001, S. 23), dann spielt es keine Rolle, an welchem Inhalt - an Goethes «Faust», einer Tageszeitung oder der Packungsbeilage eines Arzneimittels - die Kompetenz entwickelt wird, ebenso wenig, ob als Lehrmethode Frontal- oder Projektunterricht eingesetzt wird. Lehrpläne können entschlackt werden und von Festlegungen, welcher Inhalt zu welcher Zeit in welchem Umfang zu vermitteln ist, frei gehalten werden. An ihre Stelle treten so genannte Kerncurricula, die allgemein gehalten sind und nur mehr die Richtziele skizzieren, die in einem Fach erreicht werden sollen (Klieme et al., 2003, S. 94ff.). Nicht zu Unrecht tituliert Höhne (2007) den Kompetenzbegriff als «Mantra neoliberaler Bildungsreformer».

Das Defizit an inhaltlicher Begründung der Bildungsstandards ist nicht nur problematisch, weil der Reform der öffentlichen Schule dadurch eine wesentliche Legitimationsgrundlage fehlt. Es ist auch problematisch, weil in einer demokratischen Gesellschaft über Schulen nicht einfach von Experten entschieden werden kann. Vielmehr braucht es Verfahren, die festlegen, wie inhaltliche Entscheidungen zustande kommen. Diese Verfahren sind aber im Falle des HarmoSProjekts unbekannt oder werden umgangen. Entschieden wird von Experten, was nur möglich ist, wenn diesen die Position von Technokraten zugewiesen wird, die allein nach zweckrationalen Kriterien entscheiden. Nicht eine Bildungstheorie leitet die Selektion der Fächer und Inhalte, sondern die Frage der Umsetzbarkeit von Standards. Gemessen wird, was sich messen lässt. Mit einer Formulierung von Neuweg (2004) könnte man sagen: Die Einführung von Standards führt nicht dazu, «... dass ... gemessen wird, was wichtig ist, sondern dass ... wichtig wird, was gemessen werden kann.»

\section{Ist Kontrolle besser als Vertrauen?}

Bisher war von Politik und Wissenschaft die Rede. Gegenüber dem Ansinnen der Politik, Erziehungswissenschaft und Bildungsforschung zum Zweck der besseren Steuerung des Schulsystems an die Kandare zu nehmen, ist auf deren Autonomie als akademische Disziplinen insistiert worden. ${ }^{13}$ Zudem ist bestritten worden, dass die Amputation der Wissenschaft um ihre theoretische und kritische Dimension dem Unterfangen der Reform des schweizerischen Schulsystems zum Vorteil gereichen wird. Nicht zur Sprache kam bisher ein dritter Akteur, der in Bildungsfragen eine wesentliche Rolle spielt: die Lehrerschaft. Welche Rolle ist ihr zugedacht? 
Der Klieme-Bericht betont, dass der Erfolg der "standardbasierten Reform» wesentlich von ihrer Akzeptanz in der Lehrerschaft abhängt: "Nur wenn es gelingt, die praktizierenden Lehrerinnen und Lehrer zu den Hauptträgern dieser Reform zu machen, nur wenn sie diese Reform als im ureigenen professionellen Selbstinteresse liegend verstehen, wird es auf lange Sicht zu einer Erneuerung und qualitativen Veränderung der Kultur des Lehrens und Lernens kommen» (Klieme et al., 2003, S. 144). Wie aber sollen die Lehrkräfte dazu kommen, sich als Akteure einer Reform zu verstehen, die sie ihrer Professionalität beschneidet?

Professionalität beinhaltet nach gängiger Auffassung die Ausrichtung eines Berufes an den Kriterien der Wissenschaft, diese aber nicht als Torso von "gesichertem Wissen» und «erwiesenen Kausalitäten» verstanden, sondern im umfassenden Sinn eines theoretisch angeleiteten Denkens und methodischen Vorgehens. Zwar nennt die EDK neben der Harmonisierung der obligatorischen Schule und dem Aufbau eines schweizerischen Bildungsmonitorings als weiteren Schwerpunkt ihres Tätigkeitsprogramms auch die Stärkung der Lehrerprofessionalität (EDK, 2006, S. 8f., 2007a, S. 1; Mangold, Rhyn \& Maradan, 2005, S. 175). Doch steht zu befürchten, dass die subsidiäre Rolle, die sie der Wissenschaft zudenkt, auch ihr Verständnis von Lehrerprofessionalität bestimmt. Lehrerinnen und Lehrer wären dann der Wissenschaft im gleichen Sinn unterworfen wie die Wissenschaft der Politik. Damit würde die Professionalität des Lehrerberufs aber nicht gestärkt, sondern geschwächt (Bullough, Clark \& Patterson, 2003; Dupriez, 2005; Heid, 2003, S. 179f.; Herzog, 2005, 2006a). ${ }^{14}$

Zur Professionalität gehört auch, dass man auf die Bedingungen der eigenen Berufsarbeit Einfluss nehmen kann. Insofern hat Professionalität mit Autonomie zu tun (Herzog, 2003). Autonomie ist eine Frage der Freiheit, in der man eine Tätigkeit ausüben kann, und zwar nicht nur im Sinne der Fähigkeit zur autonomen Berufsausübung, sondern auch im Sinne des Freiraums, den die Gesellschaft einer Profession einräumt. Das Zugeständnis von Freiheit setzt Vertrauen voraus. Vertrauen - das wissen wir seit Lenin - ist die Alternative zu Kontrolle. Vertrauen ist in Berufen, die mit Menschen zu tun haben, unabdingbar. Mit Blick auf das amerikanische System der rigiden Handhabung von Bildungsstandards bemerken Sheldon und Biddle (1998): «... teachers should be given the same types of trust and respect we give to other professionals» (S. 177). Es wäre wünschenswert, wenn wir in der Schweiz rechtzeitig erkennen könnten, dass hier der bessere Weg der Schulreform liegt als in der Instrumentalisierung der Wissenschaft und in der Ausweitung der behördlichen Kontrolle über die Lehrerinnen und Lehrer. 


\section{Anmerkungen}

1 Da das Konkordat eine Verlängerung der Schulpflicht unter Vorverlegung des Schuleintritts um zwei Jahre stipuliert, geht es genau genommen um das vierte, achte und elfte Schuljahr (EDK, 2006).

2 «C'est de la recherche que relèvent la collecte et le traitement des données, et c'est elle aussi qui tire les conclusions qui s'imposent du point de vue scientifique», heisst es in Nummer 5 der EDK-Zeitschrift "Éducationch» (2003, S. 3f.).

3 Das erinnert an Skinner und das Prinzip der operanten Konditionierung. Skinner passt bestens in den Rahmen der aktuellen Bildungspolitik, weil auch er glaubte, auf Theorien verzichten zu können. Wie die EDK anzunehmen scheint, das schweizerische Bildungssystem lasse sich führen, wenn man es sichtbar macht (EDK, 2007a, S. 1), war Skinner (1972) der Ansicht, nie etwas anderes getan zu haben, als Situationen zu arrangieren, in denen er sehen konnte, was der Fall ist: «... we make important temporal aspects of behavior visible. Once this has happened, our scientific practice is reduced to simple looking. ... When we have achieved a practical control over the organism, theories of behavior lose their point» (S. 117, 120 - zweite Hervorh. W.H.). Die Fähigkeit zur Kontrolle ersetzt die Notwendigkeit der Theorie!

4 http://www.iso.org/iso/en/aboutiso/introduction/index.html

5 Argumentiert wird mit einem Ladenhüter der Bildungsforschung: den Studien, die Ingenkamp (1995) Anfang der 1970er Jahre zusammengestellt und kommentiert hatte. Dabei handelt es sich vorwiegend um quasi-experimentelle Untersuchungen der Beurteilungspraxis von Lehrkräften, deren ökologische Validität mehr als fraglich ist. Dass metrische Tests per se besser sein sollen, lässt sich mit diesen Studien nicht belegen. Angezeigt wäre sowieso das Gegenteil: ein Wechsel von einer «testing culture» zu einer "assessment culture» (Dochy, 2005). Dass Bildungsstandards kaum helfen, diesen Wechsel herbeizuführen, braucht nicht extra erläutert zu werden.

6 Daran ist die EDK allerdings nicht unschuldig, wie schon ihre Äusserungen zur Unzuverlässigkeit der Lehrerbeurteilung zeigen (EDK, 2006, S. 24ff.). Zudem schreibt die EDK, der den Standards zugrunde liegende Referenzrahmen werde auch «für die Entwicklung bzw. Anpassung von Instrumenten zur individuellen Standortbestimmung der Schülerinnen und Schüler verfügbar sein» (ebd., S. 23). Wenn dies noch relativ offen formuliert ist, so kann man ein paar Seiten später lesen, die «Genauigkeit» (!) der Kompetenzmodelle werde es erlauben, «durch standardisierte Tests die Schülerbeurteilung zu verbessern» (ebd., S. 26).

7 Ich behaupte nicht, die «Evidenz» dieser Studien sei nicht bestreitbar. Damit würde ich meiner eigenen Argumentation widersprechen. So gibt es zur Studie von Amrein und Berliner (2002) Gegenstimmen, auf die ich hier aber nicht eingehen kann. Aber gerade diese Gegenstimmen sowie die Reaktion von Berliner (Nichols, Glass \& Berliner, 2006) zeigen, wie verfehlt die Auffassung ist, die Wissenschaft könne der Politik so etwas wie reine Fakten liefern: «... evidence alone never tells the story» (Shulman, 2005, S. 36).

8 Besonders fatal ist, dass die Lehrkräfte ihren Unterricht nicht an den Standards, sondern an den Tests ausrichten, welche die Standards überprüfen sollen (Herman, 2004). Allerdings ist dies nichts Neues, denn schon immer haben sich die Lehrerinnen und Lehrer nicht an den Zielen des Unterrichts (Lehrplänen) orientiert, sondern an den (Lehr-)Mitteln, die ihnen verfügbar sind.

9 Für weiterführende Analysen vgl. auch Herzog 2005, 2006a, 2006b, 2007a, 2007b.

10 Keller (2002) bemerkt völlig zu Recht, Standards würden suggerieren, «dass das Technologiedefizit in der Pädagogik doch überwunden werden könnte» (S. 26).

11 Vergessen wir nicht, dass PISA ein Kind der OECD, der «Organisation für wirtschaftliche Zusammenarbeit und Entwicklung», ist und nicht von einer Organisation für Bildung und Erziehung (wie etwa der UNESCO oder UNICEF) getragen wird. Vgl. auch Fn. 13.

12 Im Extrem kehrt sich das Begründungsverhältnis um. Es sind dann nicht mehr die Inhalte, 
die den Stoff definieren, der in der Schule vermittelt wird, sondern die Kompetenzmodelle geben vor, welche Inhalte in die Lehrpläne aufzunehmen sind: Gemäss Huber (2006) ist es Aufgabe der Lehrplanarbeit, «den HarmoS-Lernzielen gute Inhalte (Lernstoffe) zuzuordnen» (S. 176). Die Messtechnik dominiert den Bildungsgedanken. Das ist es genau, was uns als kritische Botschaft aus den USA erreicht: «Testing drives the curriculum» (Paris, 2000, S. 11).

13 Einer gesonderten Diskussion bedürfte der von der CORECHED in Auftrag gegebene Bericht der OECD (2007a) über die «Educational R\&D» in der Schweiz, der die Politisierung der Bildungsforschung weiter vorantreibt. Das für den Bericht zeichnende "Center for Educational Research und Innovation (CERI)» sieht die Aufgabe der Wissenschaft «in the provision of evidence for decision-making at a policy level» (ebd., S. 3). Weiter heisst es: «... policy makers are in need of outcome-oriented, system-level studies based on quantitative methods, in order to be able to provide evidence-based policy-making» (ebd., S. 34). Man glaubt zu wissen, wessen Stimme hier spricht. Wenn man zudem weiss, welche institutionellen und personellen Verflechtungen den Bericht ermöglicht haben, dann kann man der Frage schwer ausweichen, ob sich hier jemand einen Bericht bestellt hat, um seine Ansicht über das Verhältnis von Politik und Wissenschaft bestätigen zu lassen. Die Schlussfolgerungen der OECD sind jedenfalls deutlich genug: «There are serious weaknesses in some areas including ... the need for improving the impact of research results on policy-making and educational practice» (ebd., S. 47). Da der Bericht auf fast jeder zweiten Seite das BBT für seine Praxis der Vergabe von Forschungsmitteln lobt, wird einem schwindlig: Soll dies die Zukunft der schweizerischen Bildungsforschung sein? Man glaube nicht, diese Art von politischer Einflussnahme sei von einmaliger Bedeutung: Die OECD hat eben einen weiteren Bericht veröffentlicht, in dem das CERI die «evidence-informed policy» zu einem ihrer neuen Projekte erklärt (OECD, 2007b). Womit man also vermehrt wird rechnen müssen, ist mit der «Erziehungswissenschaft der OECD» (Radtke, 2003), einer Disziplin, der die Zähne gezogen wurden und die sich als genügsames Vehikel einer ökonomisch motivierten Reformpolitik verwenden lässt.

14 Insofern lässt das Ansinnen, über das allerdings noch keine Klarheit zu bestehen scheint, aufhorchen, auch für den Lehrerberuf Standards zu entwickeln, die dann «Professionsstandards" genannt werden (EDK, 2007b, S. 17f.).

\section{Literatur}

Aldrich, R. (2000). Educational Standards in Historical Perspective. In H. Goldstein \& A. Heath (Eds.), Educational Standards. Proceedings of the British Academy (pp. 39-56). Oxford: Oxford University Press.

Amrein, A. L. \& Berliner, D. C. (2002). High-Stakes Testing, Uncertainty, and Student Learning. Education Policy Analysis Archives, 10 (18). Retrieved february 20, 2006, from http://epaa.asu.edu/epaa/v10n18/

Berz, P. (2001). 08/15. Ein Standard des 20. Jahrhunderts. München: Fink.

Böhme, G. (1993). Am Ende des Baconschen Zeitalters. Studien zur Wissenschaftsentwicklung. Frankfurt a.M.: Suhrkamp.

Böttcher, W. (2006). «Standard-Based Reform» oder: Kann man für die Schulreform von den USA lernen? In F. Eder, A. Gastager \& F. Hofmann (Hrsg.), Qualität durch Standards? Beiträge zum Schwerpunktthema der 67. Tagung der AEPF (S. 71-84). Münster: Waxmann.

Brügelmann, H. (2004). Kerncurricula, Bildungsstandards und Leistungstests. Zur unvergänglichen Hoffnung auf die Entwicklung der guten Schule durch eine Evaluation «von oben». Vierteljahrsschrift für wissenschaftliche Pädagogik, 80, 415-441.

Bullough, R. V., Jr., Clark, D. C. \& Patterson, R. S. (2003). Getting in Step: Accountability, Accreditation and the Standardization of Teacher Education in the United States. Journal of Education for Teaching, 29, 35-51. 
Dochy, F. (2005). «Learning Lasting for Life` and ‘Assessment`. How Far Did We Progress? Presidential Address at the 20th Anniversary of the European Association for Research on Learning and Instruction (EARLI), Nicosia, Cyprus, August 23.

Dubs, R. (1996). Schule, Schulentwicklung und New Public Management. Schweizerische Zeitschrift für kaufmännisches Bildungswesen, 90, 114-179.

Dupriez, V. (2005). Régulation et pilotage des systèmes scolaires: Les voies de l'harmonisation dans deux systèmes scolaires historiquement décentralisés, l'Angleterre et la Belgique francophone. Revue suisse des sciences de l'éducation, 27, 37-52.

EDK [Schweizerische Konferenz der kantonalen Erziehungsdirektoren] (2004). HarmoS. Zielsetzungen und Konzeption. Bern: EDK.

EDK [Schweizerische Konferenz der kantonalen Erziehungsdirektoren] (2006). Interkantonale Vereinbarung über die Harmonisierung der obligatorischen Schule. HarmoS-Konkordat. Bericht zur Vernehmlassung. Bern: EDK.

EDK [Schweizerische Konferenz der kantonalen Erziehungsdirektoren] (2007a). Tätigkeitsprogramm der EDK. Bern: EDK.

EDK [Schweizerische Konferenz der kantonalen Erziehungsdirektoren] (2007b). Jahresbericht 2006. Bern: EDK.

Elmore, R. E. (2004). Conclusion: The Problem of Stakes in Performance-Based Accountability Systems. In S. H. Fuhrman \& R. F. Elmore (Eds.), Redesigning Accountability Systems for Education (pp. 274-296). New York: Teachers College Press.

Fend, H. (1994). Was ist eine gute Schule? In K.-J. Tillmann (Hrsg.), Was ist eine gute Schule? (2. Aufl., S. 14-25). Hamburg: Bergmann und Helbig.

Günther, G. (1991). Idee und Grundriss einer nicht-Aristotelischen Logik. Die Idee und ihre philosophischen Voraussetzungen (3. Aufl.). Hamburg: Meiner.

Hargreaves, A. (2003). Teaching in the Knowledge Society. Education in the Age of Insecurity. Maidenhead: Open University Press.

Heid, H. (2003). Standardsetzung. In H.-P. Füssel \& P. M. Roeder (Hrsg.), Recht-Erziehung - Staat. Zur Genese einer Problemkonstellation und zur Programmatik ihrer zukünftigen Entwicklung (S. 176-193). Weinheim: Beltz.

Herman, J. L. (2004). The Effects of Testing on Instruction. In S. H. Fuhrman \& R. F. Elmore (Eds.), Redesigning Accountability Systems for Education (pp. 141-166). New York: Teachers College Press.

Herzog, W. (2002). Zeitgemässe Erziehung. Die Konstruktion pädagogischer Wirklichkeit. Weilerswist: Velbrück.

Herzog, W. (2003). Im Clinch widersprüchlicher Erwartungen. Autonomie und Professionalität in der Lehrerfortbildung. In Akademie für Lehrerfortbildung Donaueschingen (Hrsg.), 25 Jahre Staatliche Akademie für Lehrerfortbildung in Donaueschingen, 1978-2003. Bilanz und Ausblick. Reflexionen, Erfahrungen und Perspektiven (S. 39-51). Donaueschingen: Staatliche Akademie für Lehrerfortbildung.

Herzog, W. (2005). Müssen wir Standards wollen? Skepsis gegenüber einem theoretisch (zu) schwachen Konzept. Zeitschrift für Pädagogik, 51, 252-258.

Herzog, W. (2006a). Bildungsstandards: Selbstverständlichkeit oder Rückfall in technokratische Illusionen? In R. Voss (Hrsg.), Wir erfinden Schulen neu. Lernzentrierte Pädagogik in Schule und Erwachsenenbildung (S. 175-181). Weinheim: Beltz.

Herzog, W. (2006b). Bildungsstandards - ein neues Instrument der Schulreform? Odgojne znanosti, 8 (11), 11-30.

Herzog, W. (2007a). Erziehung als Produktion. Von der anhaltenden Verführbarkeit des pädagogischen Denkens durch die Politik. In C. Crotti, Ph. Gonon \& W. Herzog (Hrsg.), Pädagogik und Politik (S. 229-259). Bern: Haupt.

Herzog, W. (2007b). Pro und Contra Bildungsstandards. Die Perspektive eines Skeptikers. In Labudde, P. (Hrsg.), Bildungsstandards am Gymnasium: Korsett oder Katalysator? (S. 5764). Bern: h.e.p. 
Heubert, J. P. \& Hauser, R. H. (Eds.). (1999). High-Stakes. Testing for Tracking, Promotion, and Graduation. Washington, D.C.: National Academy Press.

Höhne, T. (2007). Der Leitbegriff ‘Kompetenz〉 als Mantra neoliberaler Bildungsreformer. Zur Kritik seiner semantischen Weitläufigkeit und inhaltlichen Kurzatmigkeit. In L. A. Pongratz, R. Reichenbach \& M. Wimmer (Hrsg.), Bildung - Wissen - Kompetenz (S. 3043). Bielefeld: Janus software Projekte.

Huber, R. (2006). Abschied vom Lehrplan -«Schlaraffenland»- weniger ist mehr. In L. Criblez, P. Gautschi, P. Hirt Monico \& H. Messner (Hrsg.), Lehrpläne und Bildungsstandards. Was Schülerinnen und Schüler lernen sollen (S. 173-181). Bern: h.e.p.

Ingenkamp, K. (1995). Zur Fragwürdigkeit der Zensurengebung. Texte und Untersuchungsberichte (9. Aufl.). Weinheim: Beltz.

Kade, J. \& Seitter, W. (2003). Jenseits des Goldstandards. Über Erziehung und Bildung unter den Bedingungen von Nicht-Wissen, Ungewissheit, Risiko und Vertrauen. In W. Helsper, R. Hörster \& J. Kade (Hrsg.), Ungewissheit. Pädagogische Felder im Modernisierungsprozess (S. 50-72). Weilerswist: Velbrück.

Keller, H.-J. (2002). Standards in der Lehrerbildung. Blick in die USA und Ausblick auf die deutschsprachige Lehrpersonenbildung. Journal für Lehrerinnen- und Lehrerbildung, 2 (1), 20-28.

Klieme, E. et al. (2003). Zur Entwicklung nationaler Bildungsstandards. Eine Expertise. Bonn: Bundesministerium für Bildung und Forschung.

Kohn, A. (2000). The Case Against Standardized Testing. Raising the Scores, Ruining the Schools. Portsmouth, N.H.: Heinemann.

Kull, M. (2007). Wissen über das Bildungswesen: Der Bildungsbericht Schweiz 2006. Gymnasium Helveticum, 61 (2), 9-11.

Lam, T. C. N. (2004). Issues and Strategies in Standards-Based School Reform: the Canadian Experience. In Th. Fitzner (Hrsg.), Bildungsstandards. Internationale Erfahrungen - Schulentwicklung - Bildungsreform (S. 103-149). Bad Boll: Evangelische Akademie.

Lind, G. (2004). Erfahrungen mit Standards in den USA - eine Übersicht. Journal für Schulentwicklung, 8 (4), 55-60.

Linn, R. L. (2000). Assessments and Accountability. Educational Researcher, 29 (2), 4-16.

Linn, R. L. (2003). Accountability: Responsibility, and Reasonable Expectations. Educational Researcher, 32 (7), 3-13.

Luhmann, N. (1984). Soziale Systeme. Grundriss einer allgemeinen Theorie. Frankfurt a.M.: Suhrkamp.

Luhmann, N. \& Schorr, K. E. (1979). Reflexionsprobleme im Erziehungssystem. Stuttgart: Klett-Cotta.

Mangold, M., Rhyn, H. \& Maradan, O. (2005). Leistungsstandards (HarmoS) und Bildungsmonitoring: zwei Hauptprioritäten der EDK und die Funktion der externen Evaluation. In G. Brägger, B. Bucher \& N. Landwehr (Hrsg.), Schlüsselfragen zur externen Schulevaluation (S. 175-185). Bern: h.e.p.

Maradan, O. (2005). Interview mit O. M. Schule \& Elternhaus, H. 1, 8-9.

Maradan, O. \& Mangold, M. (2005). Bildungsstandards in der Schweiz: Das Projekt HarmoS. ph-akzente, 12 (2), 3-7.

Marston, G. \& Watts, R. (2003). Tampering With the Evidence: A Critical Appraisal of Evidence-Based Policy-Making. Australian Review of Public Affairs, 3 (3), 143-163. Available: www.australianreview.net/journal/v3/n3/marston_watts.html [4th july 2007].

McNeil, L. N. (2000). Contradictions of School Reform. Educational Costs of Standardized Testing. New York: Routledge.

Neuweg, G. H. (2004). Bildungsstandards in Österreich. Über die gute Absicht, die Vereinbarkeit von Einsicht und Aufsicht und die gebotene Vorsicht. Pädaktuell, 4 (2), 4-13. Zugriff am 9. Juli 2007 http://www.wipaed.jku.at/mitarb/Neuweg/Neuweg_Standards_Paedaktuell.pdf 
Nichols, S. L., Glass, G. V. \& Berliner, D. C. (2006). High-Stakes Testing and Student Achievement: Does Accountability Pressure Increase Student Learning? Education Policy Analysis Archives, 14 (1). Retrieved march 12, 2007, from http://epaa.asu.edu/epaa/v14n1/v14n1.pdf

O’Day, J. A. (2004). Complexity, Accountability, and School Improvement. In S.H. Fuhrman \& R.F. Elmore (Eds.), Redesigning Accountability Systems for Education (S. 15-45). New York: Teachers College Press.

OECD [Organisation für wirtschaftliche Zusammenarbeit und Entwicklung] (2001). Lernen für das Leben. Erste Ergebnisse von PISA 2000. Paris: OECD.

OECD [Organisation for Economic Co-Operation and Development] (2007a). National Review of Educational R\&D: Switzerland. Retrieved march 27, 2007, from http://www.coreched.ch/publikationen/experts_report_en.pdf

OECD [Organisation for Economic Co-Operation and Development] (2007b). Evidence in Education. Linking Research and Policy. Paris: OECD.

Oelkers, J. (2004). Zum Problem von Standards aus historischer Sicht. Neue Sammlung, 44, 179-200.

Orfield, G. \& Kornhaber, M. L. (Eds.). (2001). Raising Standards or Raising Barriers? Inequality and High-Stakes Testing in Public Education. New York: The Century Foundation Press.

Paris, S. G. (2000). Trojan Horse in the Schoolyard. The Hidden Threats in High-Stakes Testing. Issues in Education, 6, 1-16.

Phillips, D. C. (2006). A Guide for the Perplexed: Scientific Educational Research, Methodolatry, and the Gold versus Platinum Standards. Educational Research Review, 1, 15-26.

Popper, K. R. (1969). Conjectures and Refutations. The Growth of Scientific Knowledge (3d ed.). London: Routledge \& Kegan Paul.

Popper, K. R. (1974). Objektive Erkenntnis. Ein evolutionärer Entwurf (2. Aufl.). Hamburg: Hoffmann und Campe.

Radtke, F.-O. (2003). Die Erziehungswissenschaft der OECD. Aussichten auf die neue Performanz-Kultur. In D. Nittel \& W. Seitter (Hrsg.), Die Bildung des Erwachsenen. Erziehungs- und sozialwissenschaftliche Zugänge (S. 277-304). Bielefeld: Bertelsmann.

Rousseau. J.-J. (1971). Emil oder Über die Erziehung. In neuer deutscher Fassung besorgt von L. Schmidts. Paderborn: Schöningh.

Senge, P. N. (1997). Die fünfte Disziplin. Kunst und Praxis der lernenden Organisation (4. Aufl.). Stuttgart: Klett-Cotta.

Sheldon, K. M. \& Biddle, B. J. (1998). Standards, Accountability, and School Reform: Perils and Pitfalls. Teachers College Record, 100, 164-180.

Shirley, D. \& Hargreaves, A. (2006). Data-Driven to Distraction. Why American Educators Need a Reform Alternative - and Where They Might Look to Find It. Education Week, 26 (6), 32-33.

Shulman, L. S. (2005). «eek Simplicity ... and Distrust It.) Educational Week, 24 (39), 36, 48.

SKBF [Schweizerische Koordinationsstelle für Bildungsforschung] (2006). Bildungsbericht Schweiz 2006. Aarau: SKBF.

Skinner, B.F. (1972). Cumulative Record. A Selection of Papers (3d ed.). New York: AppletonCentury-Crofts.

Specht, W. (2006). Von den Mühen der Ebene. Entwicklung und Implementation von Bildungsstandards in Österreich. In F. Eder, A. Gastager \& F. Hofmann (Hrsg.), Qualität durch Standards? Beiträge zum Schwerpunktthema der 67. Tagung der AEPF (S. 13-37). Münster: Waxmann.

Thorndike, E. L. (1921). Measurement in Education. Teachers College Record, 22, 371-379.

Schlagworte: Bildungsforschung, Bildungspolitik, Bildungsstandards, Evidencebased Policy, Lehrerprofession, Schulreform. 
